\title{
On the use of a bias-light correction for trapping effects in photoconductance-based lifetime measurements of silicon
}

\author{
Daniel Macdonald ${ }^{\mathrm{a})}$ \\ Center for Sustainable Energy Systems, Department of Engineering, FEIT, Australian National University, \\ Canberra, ACT 0200, Australia \\ Ronald A. Sinton \\ Sinton Consulting Inc., 1132 Green Circle, Boulder, Colorado 80305 \\ Andrés Cuevas \\ Center for Sustainable Energy Systems, Department of Engineering, FEIT, Australian National University, \\ Canberra, ACT 0200, Australia
}

(Received 18 September 2000; accepted for publication 7 December 2000)

\begin{abstract}
The effectiveness of a method for analytically reducing the effect of trapping centers on photoconductance-based recombination lifetime measurements in silicon is examined. The correction method involves the use of a "bias-light" term to subtract out the underlying photoconductance due to the traps. The technique extends, by approximately an order of magnitude, the range of carrier densities over which reasonably accurate (within 30\%) measurements of the recombination lifetime can be made. Guidelines for determining which bias-light intensity will produce the best correction for solar grade multicrystalline silicon wafers, and the range over which it is valid, are developed for several practical cases. (c) 2001 American Institute of Physics. [DOI: $10.1063 / 1.1346652$ ]
\end{abstract}

\section{INTRODUCTION}

Effective carrier recombination lifetime measurements are becoming increasingly valuable for solar cell characterization, both in industrial settings and in research laboratories. They allow electronically poor wafers to be discriminated and removed from process lines at an early stage. The impact of various stages of a cell process can also be monitored, and information on expected cell performance can be obtained before metallization. Injection-level dependent lifetime data are also widely utilized for determining emitter saturation current densities ${ }^{1,2}$ and, more recently, properties of surface and bulk recombination centers. ${ }^{3-5}$

However, such usefulness is contingent upon reliable measurement of the effective recombination lifetime in the injection range of interest. Photoconductance-based measurement techniques, namely microwave-detected photoconductance decay $(\mu \mathrm{W}-\mathrm{PCD})$ and inductively detected quasisteady-state photocoductance (QSSPC), are currently the only widely used methods able to determine lifetimes under low-, mid-, and high-injection conditions. Photoconductance-based methods are, however, subject to the effects of minority carrier trapping centers. ${ }^{6,7}$ When such traps are present, they can cause a significant relative excess of majority carriers, and consequently a massively distorted photoconductance. The resulting "apparent' lifetime does not represent the recombination lifetime, and hence does not correlate with final cell parameters such as open-circuit voltage, short-circuit current, or fill factor. Surface photovoltage (SPV) diffusion length measurements, on the other hand, are not subject to trapping effects, precisely because they rely on

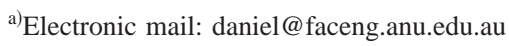

measurement of the voltage across an induced junction at the surface. Due to the delicate nature of this induced junction, however, SPV measurements are restricted to very lowinjection conditions, which are far removed from those under which a solar cell operates. Free-carrier absorption techniques, which can measure under mid- and high-injection conditions, are also subject to trapping effects.

It would be useful then to be able to mitigate the effect of trapping centers, either physically or analytically, in order to extend the range of useful data obtainable using the $\mu \mathrm{W}-$ PCD and QSSPC techniques. This is particularly relevant considering that many modern photovoltaic materials have been reported to contain minority carrier traps. These include cast multicrystalline silicon $(\mathrm{mc}-\mathrm{Si}){ }^{7}$ silicon ribbons, ${ }^{8}$ CdTe, ${ }^{9}$ and to a small extent Czochralski silicon. ${ }^{5}$ Often, the density of trapping centers in such material is large enough to affect lifetime measurements at the carrier densities corresponding to the maximum-power voltage of the finished solar cells, which is the most relevant lifetime figure of merit for cell performance. Recently, a method has been proposed which corrects, at least partially, for the effects of traps on QSSPC data. ${ }^{10}$ The correction involves adjusting injectionlevel dependent lifetime data, through the use of a "biaslight" term, in order to extend the range of usable data into lower carrier densities. The improved data potentially provide more accurate knowledge of lifetimes near cell operating conditions, and hence, allow more accurate process monitoring. The purpose of this article is to examine the efficacy of this correction method and to determine guidelines for its proper application. This is achieved by examining the effect of the correction on modeled lifetime curves 


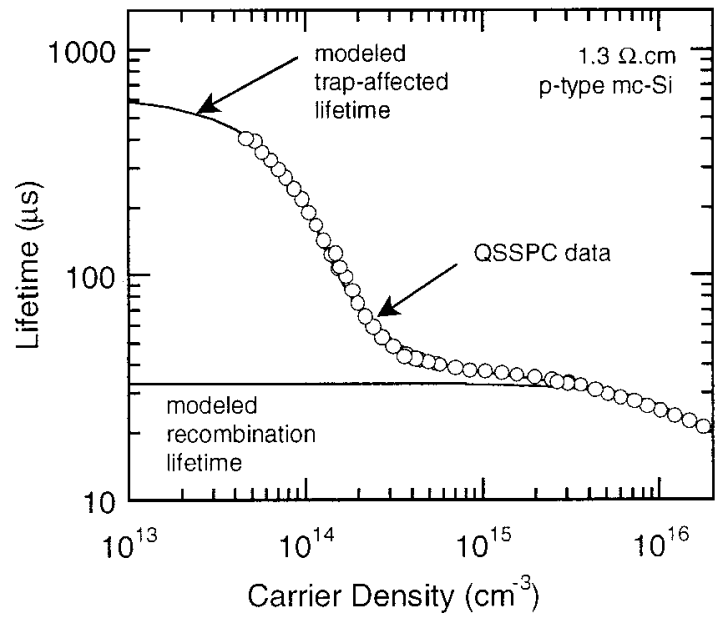

FIG. 1. QSSPC apparent lifetime measurements on a $1.3 \Omega \mathrm{cm} \mathrm{mc-Si}$ sample. Also shown are modeled recombination and trap-affected lifetime curves.

and verifying the conclusions with experimentally measured QSSPC lifetime data.

Figure 1 illustrates a typical QSSPC trap-affected (or apparent) lifetime measurement in commercially cast 1.3 $\Omega \mathrm{cm} p$-type mc-Si. This wafer has a thickness of $300 \mu \mathrm{m}$ and contains an oxide-passivated phosphorus diffused emitter on both sides characterized by a combined emitter saturation current density of $J_{O e}=2.4 \times 10^{-13} \mathrm{~A} \mathrm{~cm}^{-2}$. At carrier densities above $3 \times 10^{15} \mathrm{~cm}^{-3}$, the measured lifetime reflects the effective recombination lifetime due to the bulk, the surfaces and the emitter. Below this carrier density, however, the measured lifetime begins to rapidly increase with decreasing carrier density, typical of minority carrier trapping. Also shown on Fig. 1 are two modeled curves: the recombination lifetime and the trap-affected lifetime.

First, the effective recombination lifetime $\tau_{r}$ is modeled using

$$
\frac{1}{\tau_{r}}=\frac{1}{\tau_{\mathrm{SRH}}}+\frac{1}{\tau_{\mathrm{emitter}}},
$$

where $\tau_{\mathrm{SRH}}$ is the Shockley-Read-Hall recombination lifetime (due to both the bulk and surfaces) ${ }^{6,11-13}$ and $\tau_{\text {emitter }}$ is the recombination lifetime imposed by the emitter. ${ }^{2}$ Due to the dominance of these mechanisms, Auger and radiative recombination are negligible in all cases considered here. The resulting injection-level dependent lifetime is then fed into a trapping model ${ }^{14}$ adjusted for the QSSPC technique, ${ }^{7}$ resulting in the trap-affected curve shown. Note that the modeled trap-affected lifetime is in good agreement with the measured QSSPC data. By fitting the trapping model, the trap density in this wafer is revealed to be $4 \times 10^{14} \mathrm{~cm}^{-3}$.

Similar modeled curves are used in the following sections to investigate the effectiveness of the bias-light correction method, which can also be easily incorporated into the modeling. The ideal result would be for the correction method to cause the trap-affected curve to fall on top of the recombination lifetime curve. Of course, it is possible to achieve this by applying the full model above, with SRH and emitter recombination, plus the trapping model, to the mea- sured data, and then "turning off" the traps. ${ }^{15}$ This is, however, very time consuming. The potential advantage of the bias-light correction is that it is very easily and quickly applicable to measured data. It should be stressed that the biaslight correction considered here does not involve physically applying a bias light during measurement, but is descriptive of the analytical data correction employed.

\section{THE BIAS-LIGHT CORRECTION TERM}

The QSSPC technique is based on the simultaneous measurement of the excess photoconductance $\Delta \sigma$ via an inductively coupled coil, and the generation rate $g_{L}\left(\mathrm{~cm}^{-3}\right)$ via a reference cell. ${ }^{16}$ The generation rate is given by

$$
g_{L}=\frac{I N_{\mathrm{ph}} f_{\mathrm{abs}}}{W},
$$

where $I$ is the light intensity in units of "suns" ( 1 sun $\left.=100 \mathrm{~mW} / \mathrm{cm}^{2}\right), N_{\mathrm{ph}}$ is the incident photon flux density under standard 1 sun illumination, and $W$ is the sample width $(\mathrm{cm})$. The quantity $f_{\text {abs }}$ is the fraction of incident photons absorbed and is a function of the particular optical properties of the sample. It accounts for optical losses due to reflection from the front surface and transmission through the wafer.

The excess carrier concentration $\Delta n$ is determined by

$$
\Delta n=\frac{\Delta \sigma}{q W\left(\mu_{n}+\mu_{p}\right)},
$$

where $q$ is the electronic charge and $\mu_{n}$ and $\mu_{p}$ the electron and hole mobilities. The effective lifetime is then given by $\tau_{\text {eff }}=\Delta n / g_{L}$. An implicit assumption in this analysis is that the excess electrons and holes contribute equally (weighted for their mobilities) to the excess photoconductance. This is not true when minority carrier traps are present. First, the fact that some minority carriers are trapped means that the majority carriers contribute more heavily to the excess photoconductance. Second, since the trapped minority carriers are unable to recombine, the concentration of majority carriers increases until a new balance between generation and recombination is attained. The result is an abnormally large excess photoconductance, driven by majority carriers, at low carrier densities. In such cases $\Delta n$ is referred to as the apparent excess carrier density.

The bias-light correction attempts to overcome this problem by effectively subtracting out the trap-induced excess photoconductance. ${ }^{10}$ The analysis is then carried out relative to this "base line" photoconductance. This is illustrated in Fig. 2, which shows a linear plot of photoconductance versus light intensity for a sample affected by trapping. The plot shows the rapid increase in photoconductance at low illumination due to trapping, and also shows the positioning of the bias-light at the point where it is just sufficient to fill the traps.

The correction involves determining the change in generation $\Delta g_{L}$ relative to the generation due to the bias-light (or traps)

$$
\Delta g_{L}=\frac{\left(I-I_{\text {bias }}\right) N_{\mathrm{ph}} f_{\mathrm{abs}}}{W} .
$$




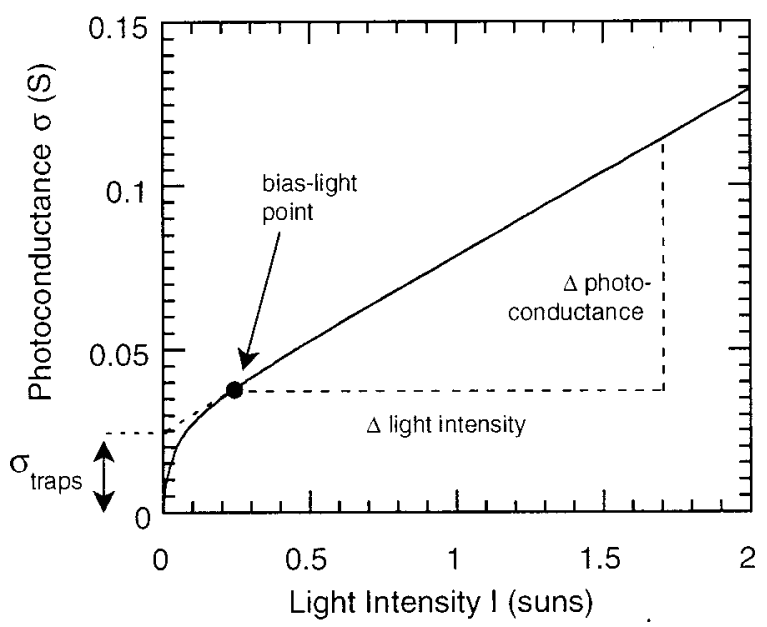

FIG. 2. Photoconductance vs light intensity for a sample affected by trapping.

The carrier density at the bias-light intensity, $\Delta n_{\text {bias }}$, can be read directly from the measured data. The corrected effective lifetime is then given by

$$
\tau_{\text {eff-corr }}=\frac{\Delta n-\Delta n_{\text {bias }}}{\Delta g_{L}},
$$

where $\Delta n$ is determined by Eq. (3). Essentially then the corrected lifetime is proportional to the ratio of $\Delta$ photoconductance to $\Delta$ light intensity, as shown on Fig. 2. Note that although the method involves subtracting out terms to account for the traps, the method is still a large-signal approach, meaning that the measured photoconductance may be much larger than the base line value, and as such the resultant corrected lifetimes reflect actual, not differential, quantities. This is appropriate for application to the QSSPC technique, which is similarly a large-signal method, unlike $\mu \mathrm{W}-\mathrm{PCD} .^{17}$

Once the corrected lifetimes are calculated, the question arises as to which carrier density should each lifetime be ascribed to. Because of the large imbalance between hole and electron populations in the trapping region, it is necessary to estimate the minority carrier density $\Delta n_{\min }$ at each data point, and plot the lifetime values against this variable, since it is the relevant parameter for solar cell operation. This is achieved by extrapolating the data from above the bias point to the photoconductance axis, as shown in Fig. 2. The value at the axis approximates the photoconductance due to the traps, $\sigma_{\text {traps }}$. The minority carrier density $\Delta n_{\min }$ is then estimated as

$$
\Delta n_{\min }=\frac{\Delta \sigma-\sigma_{\text {traps }}}{q W\left(\mu_{n}+\mu_{p}\right)} .
$$

The fully corrected data are plotted in the form of $\tau_{\text {eff-corr }}$ vs $\Delta n_{\text {min }}$. The following section examines the effect of this correction method on modeled trap-affected lifetime curves. In all cases, the recombination lifetimes and corrected lifetimes are plotted against minority carrier density $\Delta n_{\min }$, whereas the trap-affected curves are plotted against apparent carrier density $\Delta n$. Bias carrier densities $\Delta n_{\text {bias }}$ are always expressed as apparent carrier densities. The discussion is divided into three distinct cases, each characterized by

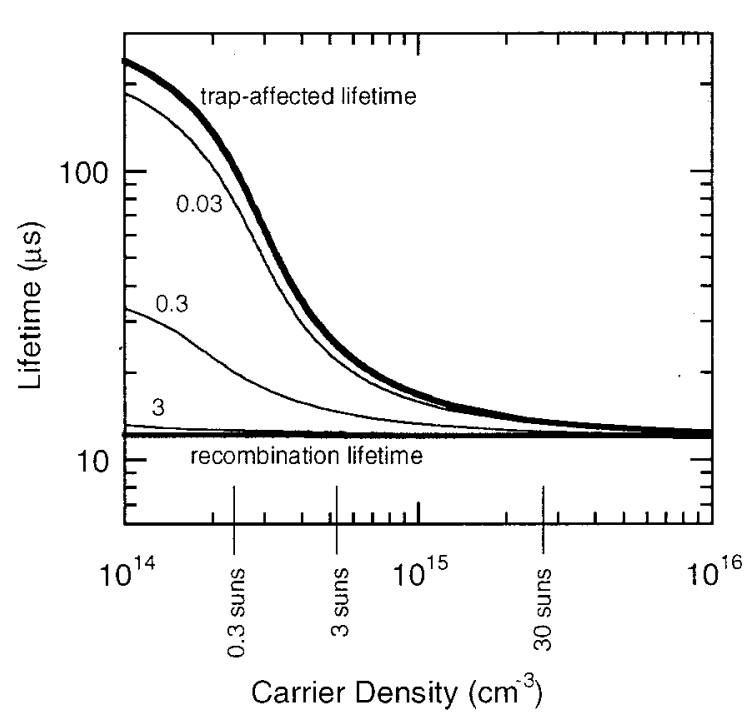

FIG. 3. Case 1: Modeled constant recombination lifetime. The recombination lifetime is $12 \mu \mathrm{s}$, and the trap density $1 \times 10^{15} \mathrm{~cm}^{-3}$. The impact of the bias-light correction is shown for bias-light intensities of $0.03,0.3,3$, and 30 suns, the latter coinciding with the recombination lifetime curve. The corresponding bias carrier densities are shown on the horizontal axis. For the 0.03 suns case the value is $5.8 \times 10^{13} \mathrm{~cm}^{-3}$.

a different injection-level dependence of the underlying recombination lifetime. It should be noted that the correction procedure sometimes gives rise to spurious asymptotic effects near the bias carrier density. These have been numerically smoothed out in the following examples.

\section{CASE 1: CONSTANT RECOMBINATION LIFETIME}

The simplest case to consider is that of an injection-level independent recombination lifetime. Such a situation is not entirely realistic, but is nevertheless commonly presumed. For our current purposes, it provides a convenient starting point for understanding the effect of the bias-light correction. Figure 3 shows the constant recombination lifetime case, with $\tau_{r}=12 \mu \mathrm{s}$, a trap density of $1 \times 10^{15} \mathrm{~cm}^{-3}$, and a trapping time to escape time ratio ${ }^{7} \tau_{t} / \tau_{g}=0.01$. Such values are typical of commercial solar grade mc-Si. Note that the trapaffected lifetime, which corresponds to that which would be measured using the QSSPC technique, is notably different from the underlying recombination lifetime even at carrier densities considerably higher than the trap density.

Also shown in Fig. 3 is the result of adjusting the trapaffected data using the bias-light correction with several different values of the bias-light intensity. On the horizontal carrier density axis, the bias carrier density is shown for each bias-light intensity. A value of 0.03 suns results in very little change in the data, since only a small fraction of the 1 $\times 10^{15} \mathrm{~cm}^{-3}$ traps are occupied, and the photoconductance is still a measure of trap filling rather than free minority carrier generation and recombination. As the bias-light intensity increases, the corrected curve more closely approximates the recombination lifetime curve. In fact, in this constant lifetime case, the larger the bias, the better the correction. At bias-light intensities above about 30 suns (giving bias carrier densities above $2 \times 10^{15} \mathrm{~cm}^{-3}$ ), the correction is close to ex- 


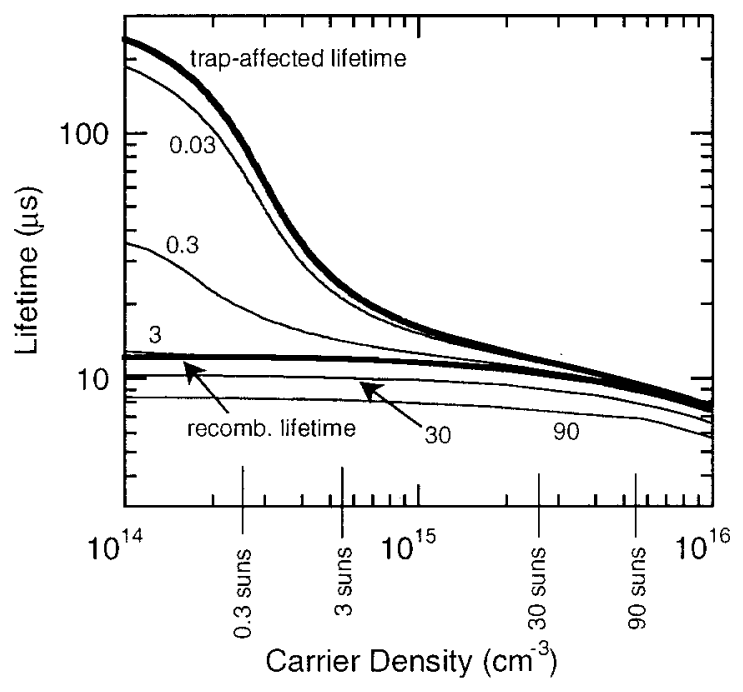

FIG. 4. Case 2: Modeled emitter recombination dominated lifetime, with $J_{\mathrm{Oe}}=2 \times 10^{-12} \mathrm{~A} \mathrm{~cm}^{-2}$. The low-injection recombination lifetime is again $12 \mu \mathrm{s}$, and the trap density $1 \times 10^{15} \mathrm{~cm}^{-3}$. The impact of the bias-light correction is shown for bias-light intensities of $0.03,0.3,3,30$, and 90 suns. The corresponding bias carrier densities are shown on the horizontal axis. For the 0.03 suns case the value is $5.8 \times 10^{13} \mathrm{~cm}^{-3}$.

act, since the bias carrier density is higher than those at which the measured lifetime is severely affected by trapping.

This illustrates an important aspect of the bias-light correction. As the bias carrier density increases, the corrected values are "based" on a point that more closely resembles the recombination lifetime at the bias carrier density. When the recombination lifetime is constant, this results in a good correction for all injection levels. However, if the recombination lifetime varies with injection level, as caused by emitter or SRH recombination, the corrected values may be based on an incorrect value - in the case of emitter recombination the lifetime will be too small at higher bias carrier densities. This is shown explicitly in the next case.

\section{CASE 2: DECREASING LIFETIME WITH INCREASING CARRIER DENSITY (EMITTER RECOMBINATION)}

Figure 4 depicts the case where the effective lifetime decreases at larger carrier densities due to recombination in the emitter, and is qualitatively similar to the data shown in Fig. 1. Such recombination is characterized by the emitter saturation current density $J_{\mathrm{Oe}}$, which in this case was chosen to have a value of $2 \times 10^{-12} \mathrm{~A} \mathrm{~cm}^{-2}$. This relatively high value was chosen for reasons discussed later, and as modeled here for a $1 \Omega \mathrm{cm}$-type substrate of thickness $300 \mu \mathrm{m}$ and $15 \%$ reflectance, results in a low-injection lifetime cap of 12 $\mu \mathrm{s}$, or, alternatively, a 1 sun $V_{\mathrm{OC}}$ cap of $603 \mathrm{mV}$. Again, a trap concentration of $1 \times 10^{15} \mathrm{~cm}^{-3}$ has been used to generate trapping effects.

The significant impact of the traps on the "measured", lifetime is obvious, and once again a bias-light intensity of 0.03 suns generates little improvement. However, the behavior for larger bias-light intensities is different to the constant lifetime case. The best correction is achieved with a bias of 3 suns. This is essentially the minimum bias required to fill the

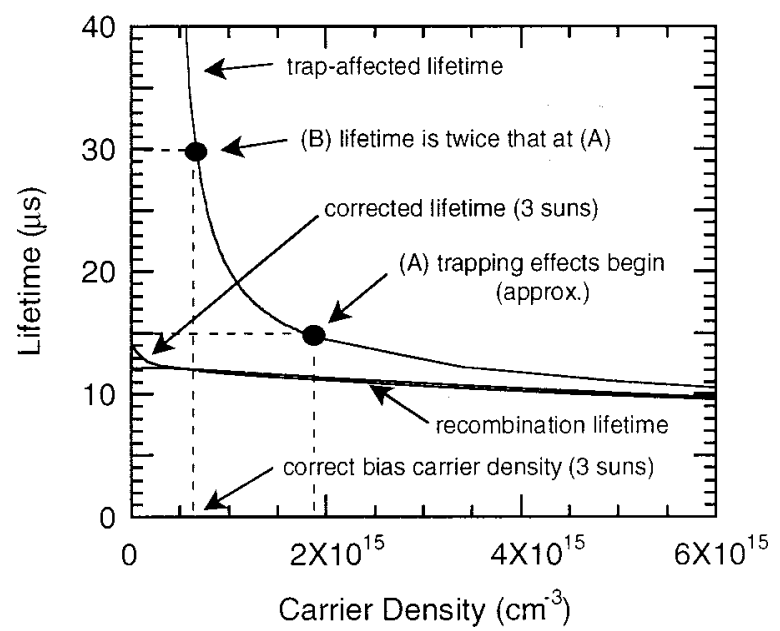

FIG. 5. Case 2: Linear horizontal axis version of Fig. 4. The method for determining the optimum bias begins by estimating, by visual inspection, the point on the trap-affected data where trapping begins to impact [point (A)]. The lifetime at this point is then doubled. The data point corresponding to this new value [point (B)] gives the bias carrier density corresponding to the optimum bias.

traps, and as such it provides the most accurate extrapolation to the axis (as shown in Fig. 2) which is necessary to estimate the minority carrier density. The figure indicates that the 3 suns correction is very good at least down to a density of $1 \times 10^{14} \mathrm{~cm}^{-3}$, which represents a significant improvement in the range of useable data available. In fact, the usable data now extends down past 1 sun open-circuit conditions, allowing much greater accuracy in monitoring cell processes and predicting device performance. For very large bias terms, such as the 30 and 90 suns cases, the bias carrier density is well above the region where trapping dominates, and consequently the extrapolation to the axis is less accurate. Furthermore, at these high carrier densities, the dependence created by the emitter recombination causes the extrapolation to reflect high-injection conditions, and is irrelevant to the low-injection region of interest. Consequently these large biases cause the corrected lifetime to underestimate the recombination lifetime in the trap-affected region.

The crucial questions then become: which is the best bias-light intensity to use (i.e., what is the minimum bias required to just fill the traps), and over what range is the ensuing corrected data accurate? From Fig. 4, it can be seen that the optimum bias of 3 suns corresponds to a bias carrier density of about $5 \times 10^{14} \mathrm{~cm}^{-3}$. In fact any bias carrier density in the range $3-7 \times 10^{14} \mathrm{~cm}^{-3}$ will give adequate results. This range straddles the carrier density at which the trapaffected lifetime is approximately twice the effective lifetime in the region just above the point where trapping begins. In other words, a good guideline for determining the best bias is to simply increase the bias until the bias carrier density is at the point where the uncorrected lifetime is approximately doubled due to the traps. This can be done easily by visually inspecting the graphed data, and is made easier by viewing a linear plot as opposed to the logarithmic one of Fig. 4. Figure 5 shows the same data on a linear scale, and the caption describes again the method for determining the optimum 
bias. Although such visual inspection is subject to uncertainty, the relative broadness of the optimum ensures that such uncertainties are small, and are more than outweighed by the ease of applying the method. An equivalent, alternative approach is to increase the bias-light until the corrected lifetime at the bias carrier density is half the uncorrected value. Care needs to be exercised with this second approach, as more than one bias-light value may satisfy the criteria. Finally, note that the properly corrected data is sufficiently accurate for all carrier densities greater than or equal to the bias carrier density.

While the accuracy of this method is independent of the magnitude of the trap density or the low-injection recombination lifetime, it does depend somewhat on the value of $J_{\mathrm{Oe}}$ since it determines the steepness of the high-injection slope. However, values of $J_{\mathrm{Oe}}$ which are less than 2 $\times 10^{-12} \mathrm{~A} \mathrm{~cm}^{-2}$ will give rise to cases with milder dependence, and in the extreme case will approach case 1 with a constant lifetime. We found that using a bias of 3 suns was very adequate also for that case, noting that a direct comparison is valid since all other parameters are the same. Consequently, we can state that this method for determining the appropriate bias level is valid for all emitters with $J_{\mathrm{Oe}} \leqslant 2$ $\times 10^{-12} \mathrm{~A} \mathrm{~cm}^{-2}$. This is a large value and should easily cover most practical industrial and laboratory cases.

As mentioned, a bias of 3 suns was also adequate for case 1 . The procedure outlined earlier for determining the optimum bias is therefore applicable to both cases 1 and 2 . The final case to consider is that in which SRH recombination centers, either in the bulk or at the surfaces, give rise to a different type of injection-level dependence.

\section{CASE 3: INCREASING LIFETIME WITH INCREASING CARRIER DENSITY (SRH RECOMBINATION)}

Figure 6 illustrates the case where SRH recombination dominates the lifetime dependence at low-to mid-injection levels. The effects of emitter recombination are retained as before, with $J_{\mathrm{Oe}}=2 \times 10^{-12} \mathrm{~A} \mathrm{~cm}^{-2}$. SRH recombination is also included, which manifests itself as an increasing lifetime as the carrier density moves from low- to mid-injection. The recombination properties of the SRH centers used were: energy level $E_{\mathrm{SRH}}=E_{C}-0.4 \mathrm{eV}$ where $E_{C}$ is the conduction band energy; electron and hole capture cross-sections $\sigma_{n}$ $=9 \times 10^{-15} \mathrm{~cm}^{2}$ and $\sigma_{p}=9 \times 10^{-17} \mathrm{~cm}^{2}$, respectively; and a volume density $N_{\mathrm{SRH}}=2 \times 10^{12} \mathrm{~cm}^{-3}$. These values are typical of interstitial metallic impurities such as iron in silicon. Once again traps were included in this case, but this time with a density reduced by an order of magnitude to 1 $\times 10^{14} \mathrm{~cm}^{-3}$, in order to allow the SRH behavior to dominate in the mid-injection range. This type of injection-level behavior is often seen in mc-Si samples with a relatively low trap density.

As in the previous cases, the lowest bias value of 0.03 suns is not sufficient to overcome the traps. As the bias is increased further, to 0.3 suns, the corrected curve moves towards the actual recombination lifetime curve, as expected. As the bias is increased still further, however, the corrected curve starts to rise again, as in the 3 and 30 sun cases. This occurs due to bias carrier density now being above the trap-

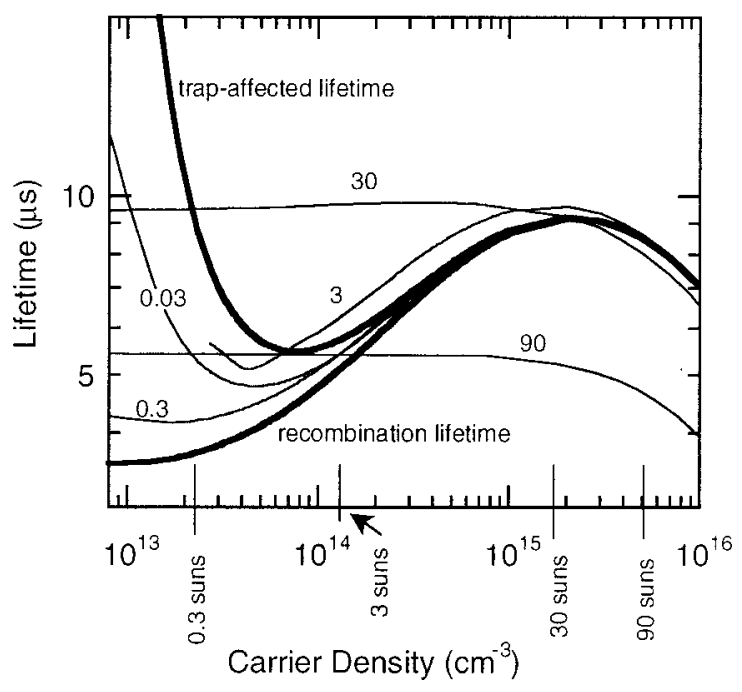

FIG. 6. Case 3: Modeled SRH recombination dominated lifetime, with $E_{\mathrm{SRH}}=E_{C}-0.4 \mathrm{eV}, \sigma_{n}=9 \times 10^{-15} \mathrm{~cm}^{2}$ and $\sigma_{p}=9 \times 10^{-17} \mathrm{~cm}^{2}, N_{\mathrm{SRH}}=2$ $\times 10^{12} \mathrm{~cm}^{-3}$ and an emitter term of $J_{\mathrm{Oe}}=2 \times 10^{-12} \mathrm{~A} \mathrm{~cm}^{-2}$. The trap density is $1 \times 10^{14} \mathrm{~cm}^{-3}$. The impact of the bias-light correction is shown for bias-light intensities of $0.03,0.3,3,30$, and 90 suns. The corresponding bias carrier densities are shown on the horizontal axis. For the 0.03 suns case the value is $9.0 \times 10^{12} \mathrm{~cm}^{-3}$.

ping region, where it is subject to the injection-level dependence of the recombination lifetime. The cause is the same as in case 2, but this time with the opposite effect due to the opposite injection-level dependence. At the highest bias level of 90 suns, the corrected data shifts back down again, since in this case the emitter recombination dominates the recombination lifetime at the bias carrier density.

Consequently, the question as to which bias level to choose for the best correction is easier to answer in this case. A good rule is to simply increase the bias carrier density from below the measurement range to the point where the low-injection data fall to their lowest point. This occurs at a bias of 0.3 suns in Fig. 6, which is an order of magnitude less than in the previous two cases, consistent with the lower trap density. Once again, the corrected data have reasonable accuracy at carrier densities greater than the bias carrier density, $2 \times 10^{13} \mathrm{~cm}^{-3}$ in this case. Specifically, the correction overestimates the recombination lifetime by about $15 \%$ at the bias carrier density, whereas the uncorrected data, at the same carrier density, overestimate by about a factor of 3 $(200 \%)$. In fact the uncorrected data only achieves an accuracy of $15 \%$ at about $1.5 \times 10^{14} \mathrm{~cm}^{-3}$, hence, the correction procedure pushes the range of usable data about an order of magnitude lower in terms of carrier densities than would otherwise be available.

\section{AN EXAMPLE: MEASUREMENTS ON $0.4 \Omega \mathrm{cm}$ mc-Si}

The previous three cases were based purely on modeled lifetime curves, although the recombination and trapping parameters used to create them are representative of standard mc-Si. In this section, real data from a $0.4 \Omega \mathrm{cm}$ mc-Si wafer are subjected to the bias-light correction procedure outlined earlier. In order to assess the accuracy of the correction, it is 


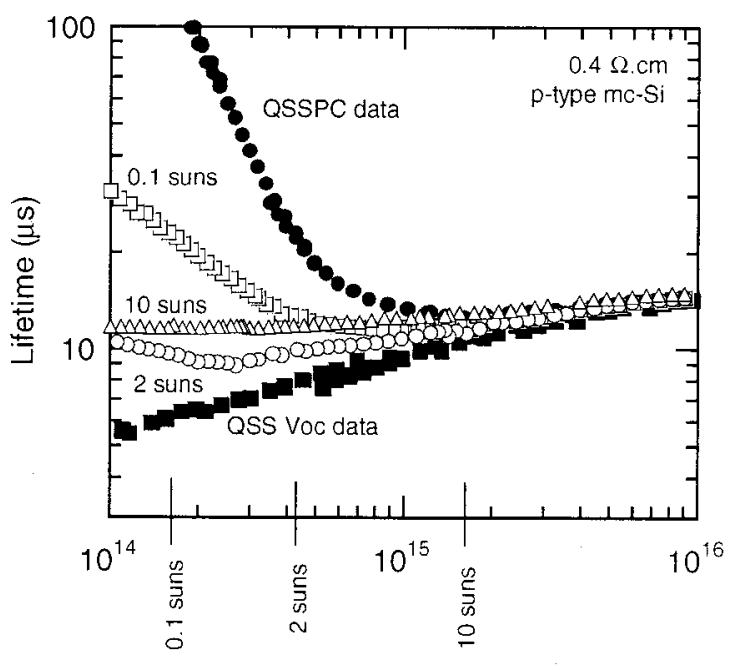

Carrier Density $\left(\mathrm{cm}^{-3}\right)$

FIG. 7. Trap-affected QSSPC lifetime and illumination- $V_{\mathrm{OC}}$ recombination lifetime measurements for a $0.4 \Omega \mathrm{cm}$ mc-Si wafer. The impact of the biaslight correction on the measured data is shown for bias-light intensities of $0.1,2$, and 10 suns. The corresponding bias carrier densities are shown on the horizontal axis.

of course necessary to have knowledge of the underlying recombination lifetime for the sample, across the whole injection-level range. This was achieved by processing the wafer into a solar cell with excellent emitter and rear surface properties, allowing the bulk to totally dominate the cells behavior. ${ }^{15}$ The illumination- $V_{\text {OC }}$ curve under quasisteadystate conditions was then measured, and the effective recombination lifetime curve inferred from the results. ${ }^{18}$ Such QSS $V_{\text {OC }}$ measurements are immune to the effects of trapping, hence, we are able to observe the recombination lifetime across a broad range of injection levels. Care needs to be taken to avoid the effect of shunts, and accurate temperature control is essential to obtain reliable recombination lifetime data with this technique, as the lifetime varies exponentially with the measured voltage, which in turn varies approximately linearly, over a small range, with temperature.

The QSS $V_{\text {OC }}$ recombination lifetime measurements are shown in Fig. 7, as well as the trap-affected lifetime curve measured by the QSSPC method prior to metallization. At carrier densities above about $4 \times 10^{15} \mathrm{~cm}^{-3}$, the two methods are in good agreement. Below this value however, the QSSPC data become increasingly inaccurate as trapping effects dominate. The QSSPC data are then corrected using the bias-light method. This sample is qualitatively like case 3, with SRH recombination apparent at low- to mid-injection, and we find that the conclusions reached in case 3 hold here also. Again, the 0.1 sun bias is insufficient to provide satisfactory correction. The 2 suns curve causes the corrected lifetime to reach its minimum at low-injection, while increasing the bias further to 10 suns causes the corrected curve to rise again. A bias-light intensity anywhere between 1 and 3 suns will give satisfactory results, corresponding to a bias carrier density range of about $3-6 \times 10^{14} \mathrm{~cm}^{-3}$. In a case such as this, the optimum bias is easy to determine, since it only needs to be increased until the corrected data reach their lowest point at the lower carrier densities, as for case 3 earlier. Note, however, that the optimum bias carrier density is very near the point where the non-trapping lifetime is doubled by the effect of traps, which is the method for determining the best bias value for case 2 situations.

The corrected data once again have significantly improved accuracy for carrier densities above the bias carrier density. In this case, the corrected and uncorrected data overestimate the recombination lifetime at the bias carrier density by about $20 \%$ and $200 \%$, respectively. Clearly, some residual SRH dependence exists in the trap-dominated part of the data and is extracted by the correction method. At carrier densities lower than the bias carrier density, however, these SRH traces are overwhelmed by the trap-induced photoconductance, and the corrected data are unreliable.

\section{CONCLUSIONS}

Use of the proposed bias-light term to correct for trapping effects in QSSPC lifetime measurements of mc-Si was found to improve the accuracy of the results in the region where trapping partially affects the measurement. Under most realistic conditions, the resulting corrected data is accurate to within about $20 \%-30 \%$ at the bias carrier density, with improving accuracy at higher carrier densities. This generally represents an extra order of magnitude of usable data, in terms of carrier densities, than would otherwise be available. This can be very significant for commercial mc-Si, which often suffers from trapping effects at the lower carrier densities corresponding to maximum-power conditions. Such an improvement in the accuracy of lower-injection lifetime data allows more accurate process monitoring. Below the bias carrier density, the corrected data may behave erratically and should be discarded. Also, asymptotic affects, arising from the correction procedure, may occur near the bias carrier density.

The central question of deciding which is the best bias value to choose can be conveniently divided into two distinct cases:

(1) Measured lifetime immediately above trapping region decreases or is constant with increasing carrier density: increase the bias until the bias carrier density is at the point where the uncorrected lifetime is approximately doubled due to the traps.

(2) Measured lifetime immediately above trapping region increases with increasing carrier density: simply increase the bias until the corrected lifetime reaches a minimum at the lower carrier densities.

Essentially these guidelines reflect the desire to use just enough bias intensity to fill the traps, but not more. Both cases can be performed by visual inspection of the QSSPC data, since the relatively broad optimum results in reasonable data for bias suns values varying by about a factor of 3. A general working principle which follows from the guidelines, and which should always be observed, is that the bias carrier density must always be less than the carrier density at which the lifetime is to be reported. If it is not possible to achieve this while observing the guidelines, then the trap density is 
too high to allow accurate correction at the desired carrier density.

\section{ACKNOWLEDGMENTS}

This work has been supported by the Australian Research Council. Thanks to Eurosolare SpA, Italy, for supplying the multicrystalline silicon wafers, and to C. Samundsett for assistance with sample preparation.

${ }^{1}$ D. E. Kane and R. M. Swanson, Proceedings of the 18th IEEE Photovoltaic Specialists Conference (IEEE, New York, 1985), p. 578.

${ }^{2}$ A. Cuevas, Sol. Energy Mater. Sol. Cells 57, 277 (1999).

${ }^{3}$ J. Schmidt, C. Berge, and A. G. Aberle, Appl. Phys. Lett. 73, 2167 (1998).

${ }^{4}$ M. Bail and R. Brendel, Proc. 16th Euro. Photovoltaic Solar Energy Conv.

Conf. (European Commision, 2000) (to be published).

${ }^{5}$ J. Schmidt and A. Cuevas, J. Appl. Phys. 86, 3175 (1999).

${ }^{6}$ J. S. Blakemore, Semiconductor Statistics, International Series of Mono- graphs on Semiconductors Vol. 3 (Pergamon, Oxford, 1962).

${ }^{7}$ D. Macdonald and A. Cuevas, Appl. Phys. Lett. 74, 1710 (1999).

${ }^{8}$ J.-W. Jeong, M. D. Rosenblum, J. P. Kalejs, and A. Rohatgi, J. Appl. Phys. 87, 7551 (2000).

${ }^{9}$ R. K. Ahrenkiel and S. Johnston, Sol. Energy Mater. Sol. Cells 55, 59 (1998).

${ }^{10}$ R. A. Sinton, Proceedings of the 9th Workshop Role of Impurities and Defects in Silicon Device Processing (NREL, Golden, CO, 1999), p. 67.

${ }^{11}$ W. Shockley and W. T. Read, Phys. Rev. 87, 835 (1952).

${ }^{12}$ R. N. Hall, Phys. Rev. 87, 387 (1952).

${ }^{13}$ D. Macdonald, M. Kerr, and A. Cuevas, Appl. Phys. Lett. 75, 1571 (1999).

${ }^{14}$ J. A. Hornbeck and J. R. Haynes, Phys. Rev. 97, 311 (1955).

${ }^{15}$ D. Macdonald and A. Cuevas, Prog. Photovoltaics 8, 363 (2000).

${ }^{16}$ R. A. Sinton and A. Cuevas, Appl. Phys. Lett. 69, 2510 (1996).

${ }^{17}$ J. Schmidt, IEEE Trans. Electron Devices 46, 2018 (1999).

${ }^{18}$ R. A. Sinton and A. Cuevas, Proc. 16th Euro. Photovoltaic Solar Energy Conv. Conf. (European Commision, 2000) (to be published). 